\title{
Preface: Celebrating the 100th anniversary of chemical sciences in Nanjing University
}

\author{
Shuhua $\mathrm{Li}^{*} \&$ Wei Wang ${ }^{*}$ \\ School of Chemistry and Chemical Engineering, Nanjing University, Nanjing 210023, China
}

Received April 10, 2020; accepted April 10, 2020; published online April 16, 2020

Citation: Li S, Wang W. Preface: Celebrating the 100th anniversary of chemical sciences in Nanjing University. Sci China Chem, 2020, 63: 561-563, https://doi. org $/ 10.1007 / \mathrm{s} 11426-020-9742-3$

The School of Chemistry and Chemical Engineering of Nanjing University was found in 1920, and it is one of the earliest departments of chemistry in China. Afterwards, the primeval National Central University's Chemistry Discipline united with Jinling University's Chemistry Discipline to form the Department of Chemistry of Nanjing University. Till 1993, the School of Chemistry and Chemical Engineering was established. The school was one of the first institutes that were approved to operate the chemistry $\mathrm{PhD}$ program in China. In 2003, 2009 and 2012, it was ranked 2nd, 3rd and 2nd, respectively, in the national disciplinary assessments organized by the Ministry of Education (MOE). In 2016, Nanjing University's Chemistry was scored A- in the disciplinary assessment. In 2017, the discipline of Chemistry as well as Chemical Engineering and Technology were both enlisted in the national "Double First-Class" initiative. Currently, the school has 169 faculty members and 62 staff members. Among them, there are 4 academicians of the Chinese Academy of Sciences, 1 adjunct academician, 14 distinguished professors and chair professors supported by the "Chang Jiang Scholars Program", 21 winners of the National Science Fund for Distinguished Young Scholars, 4 chief scientists of the National Program on Key Basic Research Project, 20 youths enrolled in the Young Overseas High-level Talents Plan and 12 winners of the National Science Fund for Excellent Young Scholars.

The school has strong atmosphere of learning, tradition of

*Corresponding authors (email: shuhua@nju.edu.cn; wei.wang@nju.edu.cn) conscientious scholarship and practical working style. There have ever been 49 academicians of the Chinese Academy of Sciences being educated and teaching here. The school was nominated as one of the first "National Basic Science Talents Training Base (Chemistry)", the "Training Plan of the National Basic Subject Top-Notch Talent" and the "National Chemical Experimental Teaching Demonstration Center". During recent years, the school has been awarded first prize once and second prizes twice of the state-level teaching awards. There are 2 teams enrolled in the State Teaching Contingents, 5 courses enlisted in the Nationally Excellent Courses and 2 enlisted in the National Excellent Open Courses. At present, the school has about 400 undergraduates and 1,100 graduates. Many students have won prizes and honors in rich competitions such as the grand prize of the "Challenge Cup" Extracurricular and Academic Contest, the China Adolescents Science \& Technology Innovation Contest, the Stars of Self-improvement of Chinese College Students and the China College Students' "Internet Plus" Innovation and Entrepreneurship Competition.

The school has the State Key Laboratory of Coordination Chemistry, the State Key Laboratory of Analytical Chemistry for Life Science, the MOE Key Laboratory of Mesoscopic Chemistry, the MOE Key Laboratory of HighPerformance Polymer Materials and Technology (B class) and the Provincial Key Laboratory of Advanced Organic Materials of Jiangsu province, etc. The chemistry building with a total area of $58,000 \mathrm{~m}^{2}$ in Xianlin Campus and a series of international advanced instruments provide strong sup- 
ports for the teaching and researching activities.

In recent years, the school has taken on and completed a number of national scientific and technologic research projects. According to the latest Essential Science Indicators (ESI), the number of papers published by the school and their total citations were ranked 21st and 29th among global chemistry research institutes, which was ranked in the global top 1\%. The US News \& World Report Best Global Universities showed that Nanjing University's Chemistry was at the 20th place in the global ranking, whereas it was ranked 28th in the ShanghaiRanking's Academic Ranking of World Universities. In the Nature Index, it was ranked 4th among global research institutes and 1st among global universities. The school has received 149 state-level and provincial-level science and technology awards including the 2nd prize of the National Natural Sciences and the 2nd prize of the National Science and Technology. The school has incubated some spin-off companies including Jiangsu Nata Opto-Electronic Material Co., Ltd. which has gone public listing in the Growth Enterprise Market of the Shenzhen Stock Exchange.

The school has been building academic exchange and cooperation with renowned international universities, research institutes and companies, successfully hosted and sponsored many international high-level academic conferences. The school has launched "Anbang Dai's Academic Lecture at Nanjing University" and "Famous Master's Lecture". Well-known international scholars were invited to give lectures and seminars at the school, which broadened the horizons of the faculty and students. The school has been establishing long-term and stable cooperation relationships with renowned international universities including University of California (Berkeley), the University of Michigan, Hokkaido University and the University of Sydney, etc., via various academic exchange programs including summer school, double major program, visiting student program and bilateral academic symposium.

Great achievements have been made in the past hundred years; on-going arduous efforts would be still needed to create new brilliance. Over the past century, the school has been upholding the motto of "be honest and intelligent, study hard and act sincerely" and the fine tradition of "be rigorous, pragmatic, creative and dedicated" to cultivate a great group of elites for the society. It has become a talenttraining and scientific research base with international influence and good reputations. To celebrate the centennial anniversary of Nanjing University's Chemistry Discipline, we organized two special issues in Science China Chemistry's Chinese and English issues, respectively. Their contents cover vast frontiers in the realm of chemistry including synthetic chemistry, single cell analysis, electrocatalysis, optoelectronic materials, ionic liquids, bio-imaging sensors, biomaterials, functional coordination materials, etc. By presenting our latest research accomplishments, we would like to sincerely thank for the great efforts made from the beloved faculty members as well as the kind concern, support and great help from all the superiors, academic colleagues and all sectors of society. Also, we hope to express our belief of "never forget why you started and move forward bravely", and our dedications to creating a new glorious era in the coming century.

The Science China Chemistry's Editor-in-Chief, Prof. Lijun Wan's enthusiastic support and Editor Xuemei Zhang's thoughtful guidance and organization are essential for these two special issues. Herein, we would like to present our sincere appreciation to their great efforts. The School of Chemistry and Chemical Engineering of Nanjing University will still carry forward the fine traditions, pioneer and innovate ceaselessly, to achieve its goal towards the international first-class discipline, and further dedicate to developing the national higher education in chemistry and promoting the progress in chemical sciences! 

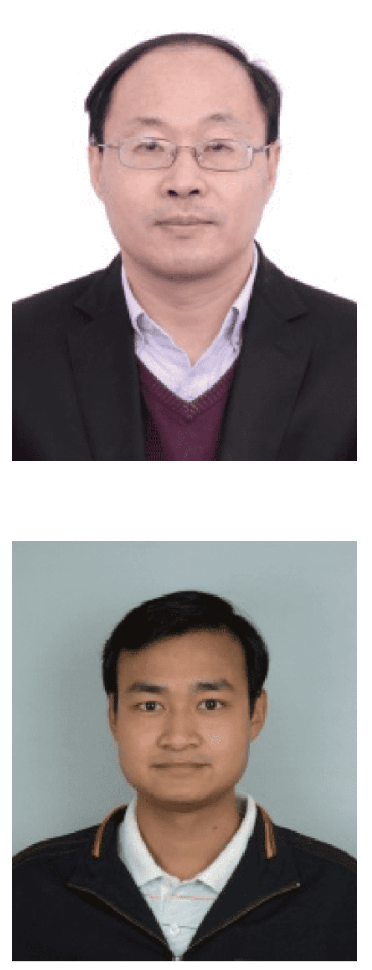

Shuhua Li is a professor and the dean of School of Chemistry and Chemical Engineering, Nanjing University. He received his BS and MS from Central South University of Technology in 1990 and 1993, respectively, and his PhD from Nanjing University in 1996. His research focuses on the development of efficient quantum chemistry methods for large systems and computational design of chemical reactions, and has made important and original contributions in the related subjects. He won the National Science Fund for Distinguished Young Scholars in 2006, and the Pople Medal of Asian Pacific Association of Theoretical \& Computational Chemists in 2008. He was appointed as Changjiang Scholar Chair Professor by Ministry of Education in 2009, and elected as a member of the International Academy of Quantum Molecular Science in 2017. He won the first prize in the Natural Science Award of Ministry of Education in 2019.

Wei Wang is a professor and vice dean of School of Chemistry and Chemical Engineering, Nanjing University. He received his BS in 2004 and PhD in 2009, both from the University of Science and Technology of China (USTC). His current researches involve advanced optical microscopy for single-cell imaging, nanoelectrochemistry, and single-molecule detection. He won the Young Chemist Award from the Chinese Chemical Society in 2017, and received the National Science Fund for Distinguished Young Scholars in 2019. 